\title{
Análise comparativa dos sistemas de saúde brasileiro e britânico na atenção básica
}

\section{Comparative analysis of Brazilian and British health systems in the Primary Health Care}

Recebido em: 05/05/2017

Aceito em: $\quad 02 / 08 / 2017$
Maria Aparecida NICOLETTI; Taissa de Mattos FARIA

Departamento de Farmácia, Faculdade de Ciências Farmacêuticas, Universidade de São Paulo. Avenida Professor Lineu Prestes, 580, CEP 05508-900.

São Paulo, SP, Brasil.E-mail: nicoletti@usp.br

\section{ABSTRACT}

Both, Unified Health System (SUS) and the National Health Service (NHS) offer free access to the population. However, regarding the development of primary health care, differences can be noted in the coverage of these two public health systems. Therefore, the objective is to carry out a critical comparative analysis of these two services. A literature review was conducted on scientific electronic databases and web pages of the Ministry of Health and United Kingdom Department of Health. The results showed although SUS and NHS are based on the same principles, they had to make different decisions over the years to adapt to the socioeconomic, epidemiological and historical several contexts. In SUS context, regarding the management and financing of the system, occurred a progressive increase in the participation of municipalities and states while the federal public budget decreased. The NHS funding, instead, remained from national taxes, and the regional offices are responsible for the execution and contracting of the health services.

Keywords: Unified Health System; health services accessibility; public health.

\section{RESUMO}

O Sistema Único de Saúde (SUS) e o National Health Service (NHS) possuem caráter público e oferecem acesso gratuito aos serviços de saúde. No entanto, em relação ao desenvolvimento da atenção básica, existem diferenças na cobertura destes dois sistemas. O objetivo foi fazer uma análise comparativa crítica entre os dois sistemas. A revisão bibliográfica do tipo narrativa foi conduzida em bases eletrônicas científicas de dados e portais do Ministério da Saúde e United Kingdom Department of Health. Apesar de serem guiados pelos mesmos princípios, o SUS e o NHS assumiram decisões diferentes ao longo dos anos a fim de se adaptarem às realidades socioeconômicas, epidemiológicas e contextos históricos que presenciaram. No SUS, foi observado um aumento progressivo da participação dos Municípios e Estados na gestão e financiamento do sistema e uma diminuição dos gastos públicos federais. No NHS, o financiamento se manteve concentrado na coleta de impostos da esfera nacional, estando as estâncias regionais responsáveis pela execução e contratação dos serviços de saúde.

Palavras-chave: Sistema Único de Saúde; acesso aos serviços de saúde; saúde pública. 


\section{INTRODUÇÃO}

Os sistemas de saúde brasileiro e inglês. A ideia de saúde como um direito vem sendo admitida desde 1946, quando a Organização Mundial de Saúde (OMS) adotou em sua constituição a saúde como direito humano fundamental, sem discriminação (1). Esse despertar para os direitos humanos, em termos de saúde pública, fez suscitar, em vários países, reformas dos sistemas de saúde no sentido de relacionar, de forma efetiva, a justiça social a saúde pública (1).

O objetivo da OMS foi que todos, inclusive os mais carentes, tenham acesso aos serviços de saúde sem a necessidade de pagamentos adicionais pelos próprios pacientes, o que gera sacrifícios financeiros para eles e a OMS acredita que o mundo está longe de atingir esta cobertura universal considerada como ideal (2).

Com relação aos modelos de gestão, nenhum país tem um modelo puro, único; porém, em alguns se observa uma grande hegemonia de determinada forma de organização e financiamento da saúde que caracteriza o modelo. Assim, quando se diz que um país adota um determinado tipo de sistema, está se falando, na verdade, do sistema que predomina naquele país (3).

Os sistemas de saúde encontrados em diferentes países apresentam elevada complexidade e a preocupação com o aporte financeiro é constante, considerando que estão diretamente relacionados ao sistema econômico do país e não estão dissociados das políticas públicas regulatórias.

O direito à saúde encontra-se relacionado a inúmeros fatores que, muitas vezes, não são previamente identificáveis, tais como avanços tecnológicos, que redundam em novos e mais eficientes tratamentos; surtos epidêmicos, que demandam imediata reação por parte do Poder Público; modificações estruturais da sociedade (como o envelhecimento da população), que exijam o repensar do planejamento sanitário. Desse modo, a relativamente baixa densidade normativa do conteúdo constitucional do direito à saúde não pode ser compreendida como baixo nível de vinculação jurídica. Ao contrário, a natureza principiológica da norma é condição para que sua realização e proteção alcancem níveis adequados, de acordo com as peculiaridades as especificidades de cada região e a partir das ações e serviços de saúde existentes em dado momento histórico (4).

Desde a Declaração dos Direitos Humanos (5) até hoje há aumento da consciência de que saúde é um direito humano, e o reconhecimento da importância desse direito para as questões econômicas e sociais, considerando saúde não somente o direito a assistência médica, mas também ao acesso, aos recursos, a aceitação de práticas culturais, e a qualidade dos serviços, ressaltando também o entendimento de que os determinantes sociais da saúde são tão ou mais importantes que os fatores ambientais e biológicos, e que precisam ser considerados na elaboração e implantação de políticas públicas de saúde, as quais devem ser integradas com outras áreas do governo de forma a torná-las amplas e mais eficientes (1).

Sistema Único de Saúde (SUS). Os ideais acerca dos cuidados de saúde pública levantados na Constituição Brasileira de 1988 (6) foram formalizados por meio da Lei n. 8.080, de 19 de setembro de 1990 (7) e Lei $n$. 8.142, de 28 de dezembro de 1990 (8) e definiram os guias para os recursos de financiamento do SUS. As políticas de financiamento do sistema buscaram promover a descentralização dos serviços de atendimento em níveis municipal e estadual, estabelecendo as responsabilidades de cada nível governamental a fim de garantir o acesso universal a todos (9). A finalidade de sua criação foi mudar a situação de desigualdade do atendimento à saúde da população e estabelecer o atendimento público aos serviços primário, secundário e terciário para qualquer cidadão que assim necessitasse (10).

No SUS, os recursos são financiados pelo governo federal e repassados para os municípios por um critério per capita. No entanto, ao longo da década de 1990, o gasto público com o SUS na esfera federal tem diminuído e a participação dos municípios no custeio do sistema tem aumentado. Progressivamente, verifica-se uma transferência federal direta para as esferas estaduais e municipais visando o financiamento das ações e serviços do SUS, passando estes a assumir maior responsabilidade sobre a gestão do sistema (11).

Dentre os países desenvolvidos, a Inglaterra apresenta um sistema de saúde universal e igualitário com atuação preventiva e curativa. Neste sentido, o sistema inglês apresenta vários aspectos semelhantes e, também, divergentes ao SUS do Brasil que serão abordados comparativamente.

National Health Service (NHS). Criado em 1948, o National Health Service (NHS), Sistema de Saúde do Reino Unido, possui caráter público e foi organizado em três componentes, ou seja, serviços hospitalares especializados, serviços qualificados como "general practitioner" e de saúde pública (12). Em sua origem, era composto por 14 Autoridades Sanitárias Regionais e três Escritórios Provinciais - País de Gales, Escócia 
e Irlanda do Norte; o NHS baseou-se nas doutrinas de universalidade, equidade e integralidade, as mesmas que guiaram a criação do sistema de saúde brasileiro, o SUS (12).

No NHS, inicialmente, havia a intenção de descentralização dos serviços e a consequente atribuição aos municípios; entretanto, houve resistência da classe médica e não foi efetivada em razão de que médicos de atenção primária recebiam por captação, isto é, conforme o número de pacientes que compunham sua lista de pacientes (12). O sistema permaneceu integralmente público e centralizado até 2004, quando houve uma separação para os quatro países que compõem o Reino Unido: Inglaterra, Escócia, País de Gales e Irlanda do Norte. Embora a base do sistema permaneça a mesma entre os países, a execução em cada país apresenta algumas peculiaridades. O NHS da Inglaterra, dos quatro países do Reino Unido, é o que mais recebe recursos do Departamento de Saúde do Reino Unido (13) e, por esta razão, será utilizado para nortear este estudo.

O financiamento, no NHS, advém do setor público por meio, principalmente, de impostos gerais (do Tesouro Nacional), com uma pequena contribuição do Sistema de Seguridade Social. O único gasto dos cidadãos britânicos no sistema público de saúde refere-se ao pagamento por prescrição medicamentosa ambulatorial. O repasse dos recursos para os diferentes municípios se dá por um critério per capita, com base de captação ponderada, ou seja, é ajustado a fatores demográficos e epidemiológicos, como idade, saúde e localização da população. Em torno de $47 \%$ do orçamento do NHS é gasto em cuidados emergenciais. Setores como clínica médica, cuidado à saúde, saúde mental e prescrições somam ao redor de $10 \%$ do total. Para a gestão do NHS, anualmente, o governo estabelece o quanto lhe será destinado em relação aos impostos recolhidos (14).

Semelhanças e diferenças gerais entre os sistemas. Apesar do alto grau de similaridade entre os dois sistemas, o investimento na atenção básica difere entre o SUS e o NHS.

No SUS, até 2012, apenas 30-40\% da população apresentavam cobertura efetiva, devido aos desafios enfrentados pelo Ministério da Saúde para alocação de investimentos na infraestrutura adequada para desenvolver a atenção básica de qualidade no Brasil $(15,16)$. Vale ressaltar aqui que, embora documentos recentes utilizem o termo "saúde básica", no Brasil os termos "saúde primária" e "saúde básica" são tidos como equivalentes $(17,18)$.
No NHS, a atenção básica de qualidade é um dos focos principais do governo, apresentando quase que a totalidade da população com acesso a atendimento primário de qualidade $(12,14)$.

O NHS também sofreu reformas após a sua criação, guiadas pelo contexto histórico ao qual ele estava inserido no momento. Porém, apesar das reformas, o sistema conseguiu preservar os princípios de universalidade, integralidade e equidade que o conceberam, o que provou que mesmo sistemas de saúde sólidos necessitam se adaptar a situação corrente da sua sociedade.

Embora em diferentes graus de desenvolvimento ambos os sistemas - SUS e NHS - apresentam princípios e características muito semelhantes, levando a crer que o NHS poderia ter servido de base para a criação do SUS (12).

Diante disto, o objetivo é proporcionar informação contextualizada decorrente da análise comparativa da estrutura dos sistemas de saúde brasileiro e inglês, considerando suas semelhanças, diferenças e os pontos de desenvolvimento com foco principal na Atenção Básica.

\section{MÉTODOS}

Revisão bibliográfica narrativa em bases científicas virtuais de dados e adicionalmente, foi realizada consulta a partir do levantamento de doutrinas, diretrizes, decretos, guias, cartilhas e manuais, por meio de pesquisa em sites específicos, como o do Ministério da Saúde e United Kingdom Department of Health.

$\mathrm{Na}$ estratégia de busca foram utilizados os descritores "SUS", "NHS", "SUS structure", "NHS structure", "Primary health care" ou permutação com ao menos dois destes. A revisão bibliográfica foi realizada em bases de dados disponíveis no Portal de Periódicos da Capes, Scientific Electronic Library Online, US National Library of Medicine - National Institutes of Health e no Portal do Sistema Integrado de Bibliotecas da Universidade de São Paulo, utilizando como critério de inclusão, abordagem diretamente relacionada ao objetivo proposto.

É importante salientar, entretanto, que a apresentação do texto, a seguir, está organizada de maneira que cada tópico discorrido aborde os seguintes aspectos relacionados ao Sistema Único de Saúde e ao National Health Service, respectivamente: a) Estrutura dos Sistemas, b) Financiamento dos Sistemas, c) Atenção Básica em Saúde e d) Acesso a Medicamentos, onde as principais diferenças e semelhanças estão resumidamente contempladas. 


\section{RESULTADOS E DISCUSSÃO}

Sistema Único de Saúde. Os princípios do SUS começaram a surgir na década de 1970 , em meio a movimentos antiditadura e da Reforma Sanitária que foram essenciais para estabelecer a diretrizes que regem o SUS até os dias atuais (15). Na década de 1970, as periferias das cidades médias e grandes presenciaram intensa migração de pessoas da zona rural e cidades pequenas em decorrência do empobrecimento destas regiões, ocasionado pela falta da reforma agrária e estatuto de terra. Neste cenário de tensão social aliado a medidas não efetivas tomadas pelas Prefeituras Municipais, como atendimentos precários à saúde, fortaleceu-se o movimento criado por jovens sanitaristas que clamava por melhores condições de saúde (15). Este movimento, liderado por profissionais e intelectuais da área da saúde, ganhou o apoio de lideranças políticas sindicais, lideranças populares, parlamentares e instituições oficiais de saúde e levantou publicamente o ideal de novo modelo de saúde para o Brasil, baseado em um sistema público, racional, universal e descentralizado. Assim, a partir da Constituição da República Federativa do Brasil de 1988 (6), a saúde veio ser estabelecida como direito de todos e um dever do Estado, dando oportunidade para o surgimento oficial do Sistema Único de Saúde (19).

Em 1990, o Ministério da Saúde lançou a primeira cartilha onde estabeleceu que o Sistema Único devia seguir a mesma doutrina e os mesmos princípios organizativos em todo o território nacional sob a responsabilidade das três esferas autônomas de governos federal, estadual e municipal, entendendo que o SUS não é serviço e nem mesmo instituição, mas sim um Sistema que significa um conjunto de unidades, de serviços e ações que interagem para um fim comum (20). Assim, foi definido que o SUS seria regido pelas doutrinas de universalidade, integralidade e equidade. Ou seja, o sistema deve atingir igualmente todas as pessoas das diferentes regiões brasileiras oferecendo acesso gratuito aos serviços em saúde. Ao mesmo tempo, o SUS foi organizado segundo os seguintes os princípios: resolutividade, descentralização, hierarquização/regionalização, participação do cidadão e complementariedade do setor privado (20).

Com o intuito de viabilizar as doutrinas e princípios do SUS, foi definido o papel de cada gestor dentro do sistema. No Brasil, o sistema político federativo adotado prevê a difusão de poder e autoridade entre as esferas de governo que o compõem. Assim, assume-se a existência de poderes de governos concorrentes dentro de um mesmo território. Este modelo de organização refletiu-se di- retamente na gestão do SUS, onde se viu a transferência de serviços, poder, responsabilidades e recursos para as instâncias estaduais e, principalmente, municipais, desconcentrando-os do governo federal (11).

Adicionalmente, as atividades sob a responsabilidade do Ministério da Saúde são executadas por sete secretarias com competências diferentes e com as funções de planejar, propiciar e efetuar as políticas de saúde. As competências destas Secretarias incluem: Secretaria de Ciência, Tecnologia e Insumos Estratégicos; Secretaria de Atenção à Saúde; Secretaria Executiva; Secretaria de Gestão Estratégica e Participativa; Secretaria de Gestão do Trabalho e da Educação na Saúde; Secretaria Especial de Saúde Indígena; Secretaria de Vigilância em Saúde (21).

O SUS consolidou-se, ao longo de duas décadas, como a maior política de Estado do País, promotor de inclusão e justiça social. O Brasil é conhecido internacionalmente como uma referência no setor da saúde por causa das iniciativas do modelo de saúde brasileiro (22).

National Health Service. Mais antigo que o SUS, o sistema nacional de saúde inglês surgiu em 1948, pautado nos princípios de igualdade, integralidade e universalidade (12). Assim como o SUS, o NHS também passou por reformas de acordo com o momento político e econômico em que o país se encontrava, mas sem abandonar os princípios que estimularam a sua criação. Ao longo dos anos 1980 e 1990, duas reformas de caráter liberalizante iniciaram a tentativa de introdução do mercado privado no sistema, porém, tais tentativas não alcançaram o resultado esperado e o sistema manteve seu caráter inteiramente público (12).

A mais recente transformação ocorrida no sistema de saúde inglês foi o lançamento do Health and Social Care Act (H\&SCA) (23). Assinado em 2012, este ato estabeleceu medidas legislativas na maneira como o NHS é organizado, as quais guiam a estrutura do sistema até hoje (14).

Uma medida significativa do H\&SCA foi a criação dos Clinical Commissioning Groups (CCG). Os CCG são órgãos legais do NHS distribuídos geograficamente e cuja função é planejar e delegar os serviços de cuidados de saúde à população da área pela qual ele é responsável. Dentre os membros dos CCG incluem os General Practioner (GP) e outros profissionais da saúde, como enfermeiros. Os CCG são responsáveis em sua maior parte pelos cuidados secundários, tais como: cuidados hospitalares planejados, reabilitação, emergências e pronto socorro, serviços para saúde mental e dificul- 
dades de aprendizado, além da maioria dos serviços de saúde para a comunidade $(14,24)$.

Apesar da descentralização da gestão em saúde com a criação dos CCG, algumas atribuições continuaram a cargo do NHS, devendo este constantemente direcionar a melhoraria da saúde e sendo diretamente responsável pelo fornecimento da atenção básica à população (24).

De acordo com o guia emitido pelo próprio NHS em 2014 (14), suas atribuições incluem:

- Saúde Primária (Atenção Básica): fornecida por uma variedade de provedores, como os GP, dentistas, optometristas, farmacêuticos e centros de pronto atendimento.

- Acute Trusts: serviços secundários e mais especializados. Estes serviços em sua maioria são providos pelos CCG, porém alguns mais especializados são providos diretamente pelo NHS.

- Ambulance Trusts: serviços de transporte emergenciais tanto para enfermidades fatais quanto não fatais. Em alguns casos, também inclui serviços não emergenciais de transporte hospitalar.

- Mental Health Trusts: prestação de serviços comunitários, internamento e assistência social para uma ampla gama de doenças psíquicas. São serviços em geral de responsabilidade dos CCG. No entanto, também podem ser encontradas em organizações do próprio NHS, no setor voluntário e no setor privado.

- Serviços comunitários de saúde: providos por fundações e não fundações comunitárias de saúde. Os serviços incluem enfermeiros locais, visitantes de saúde, escola de enfermagem, serviços especializados para a comunidade, hospital domiciliar, centros de pronto-socorro e reabilitação domiciliar.

Além das atribuições estabelecidas, outra importante decisão surgida no H\&SCA foi a criação legislativa da Public Health England (PHE), uma agência executiva inserida no Departamento de Saúde do Reino Unido (DH) para tratar de questões de saúde pública. A PHE tem como objetivo proteger e melhorar a saúde da nação e direcionar esforços para tratar das desigualdades em saúde (14).

A nova legislação prevê, também, que a responsabilidade de monitoramento e da regulação do sistema seja compartilhada por um conjunto de diferentes grupos com a finalidade de garantir que saúde de qualidade seja fornecida para a população. Dentre estes diferentes grupos, dois exemplos importantes são os Care Quality Commission (CQC) e o os Healthwatch England $(14,24)$. Os CQC são reguladores independents, cuja função é assegurar a qualidade dos serviços sociais e de saúde. Eles inspecionam hospitais, casas de cuidado, clínicas médicas (comandadas pelos GP), clínicas odontológicas e outros. Se os serviços prestados não estiverem dentro dos padrões de qualidade e segurança exigidos, os CQC têm o poder de advertir, penalizar, suspender, cancelar ou restringir os serviços prestados pelo estabelecimento ou, ainda processá-lo (14).

Os Healthwatch England, por sua vez, são organizações independentes existentes em todas as autoridades locais do NHS com intuito de defender os interesses da população. Foram criadas a fim de direcionar o envolvimento direto da população na saúde pública, possuindo voz legal perante aos que regulam e executam as decisões na saúde $(14,25)$.

Por fim, com a finalidade de manter e padronizar as ações em saúde, o H\&SCA conferiu ao National Institute for Health and Care Excellence (NICE) a função de desenvolver guias e padrões de qualidade e ao Health and Social Care Information Centre (HSCIC) a responsabilidade de coletar, analisar e apresentar dados a de saúde e cuidados sociais (14).

\section{FINANCIAMENTO DOS SISTEMAS}

Sistema Único de Saúde. Tanto no SUS quanto no NHS, o financiamento advém do setor público por meio da coleta de impostos da população. Em ambos os casos, a população tem direito a acesso aos serviços de saúde sem custos adicionais. Esta forma de financiamento reflete os princípios de universalidade, integralidade e igualdade comuns aos dois sistemas $(13,26)$.

No entanto, apesar de muito semelhantes, algumas diferenças entre os dois sistemas podem ser evidenciadas. Segundo dados da OMS, o Brasil possui população absoluta de aproximadamente 207 milhões de pessoas, possuindo gasto total em saúde de $8,3 \%$ (27). O Reino Unido, por sua vez, possui população de cerca de $64 \mathrm{mi}-$ lhões, com gasto total em saúde de $9,1 \%$, sendo que $87 \%$ destes gastos são direcionados somente para a Inglaterra, que apresenta uma população de aproximadamente 54 milhões de pessoas (27). Assim, apesar de possuírem gastos equivalentes, o SUS necessita cobrir uma população quase quatro vezes maior que o NHS inglês, o que reflete no gasto per capita em saúde do país, no qual é 2,5 vezes maior no NHS do que no SUS $(24,27)$. 
Adicionalmente, segundo Lottemberg (2015), um ranking comparativo do grau de eficiência dos sistemas de saúde realizado pela OMS mostrou que, entre 191 países analisados, países com produto interno bruto (PIB) menor do que o Brasil, ficaram melhor classificados (28). Dentre estes podem ser citados, na América Latina, Colômbia, Chile, Costa Rica e Cuba. O Brasil ocupou a $125^{\text {a }}$ colocação no ranking total. De modo geral, para a OMS, um Sistema de Saúde exitoso deve se sustentar em sua capacidade de melhorar a saúde da população, ter capacidade de responder aos anseios de seus usuários e ser financeiramente viável. Atualmente, o Brasil gasta proporcionalmente muito menos que gastava há dez anos (28).

A Constituição da República Federativa do Brasil de 1988 (6) estabeleceu que os fundos para o financiamento do SUS viriam do Orçamento de Seguridade Social, de onde $30 \%$ deveriam ser destinados diretamente à saúde até que as diretrizes orçamentárias fossem aprovadas, excluindo-se o seguro-desemprego. Na prática, este repasse não ocorreu, fazendo com que o SUS vivenciasse crises financeiras durante a década de 1990. Neste contexto, surgiram várias Emendas Constitucionais (EC) com intuito de reverter este quadro econômico. Nos anos 2000 foi criada a EC n ${ }^{\circ} 29 / 2000$ (29), a qual criou regras para a participação no custeio do SUS das esferas federais, estaduais e municipais. Oficializava-se assim, o processo de descentralização do financiamento do SUS, dando início a um aumento progressivo da participação estadual e municipal no custeio do Sistema, aliado a uma diminuição da participação federal. Entre os anos 2002 e 2014, houve uma redução de 52\% para $42 \%$ da participação da União no financiamento do SUS, e um aumento de $23 \%$ para $26 \%$ da participação estadual e de $25 \%$ para $31 \%$ da municipal, comprovando este cenário de descentralização no financiamento do SUS $(11,19,26)$.

No SUS, pode ser observada a necessidade de atender uma população muito grande com perfis sociais e epidemiológicos próprios. As diferenças regionais encontradas no País levaram à consolidação de um sistema descentralizado, com gestores direcionados a exercer políticas em saúde que atendam às necessidades da sua população. No entanto, o despreparo e a falta de qualificação dos gestores locais para exercer políticas públicas fazem com que os programas nem sempre apresentem bons resultados e os serviços em saúde fiquem prejudicados (30) .

Neste sentido, um dos exemplos que caracteriza falha de gestão está, por exemplo, relacionado à aqui- sição de medicamentos. Estima-se que R 1 bilhão em medicamentos é desperdiçado todo ano devido a grandes compras de estoque, controle inadequado, armazenamento incorreto e má distribuição (31). Ainda, o modelo de política estabelecido muitas vezes não leva em consideração o tempo de mandato de cada gestor, fazendo com que certas políticas não tenham continuidade.

O financiamento descentralizado do SUS, aliado à falta de conhecimentos político-econômicos de muitos gestores locais, fazem com que os recursos nem sempre sejam alocados de maneira adequada, gerando situações de desigualdades em saúde. Ainda, a progressão das ações judiciais no SUS indica provável incapacidade do sistema em dispor de recursos financeiros para atender a necessidade de medicamentos da Assistência Farmacêutica, uma vez que o valor disponibilizado constitui um porcentual fixo do que é arrecadado pela União (32).

National Health Service. Na Inglaterra, assim como no Brasil, ocorreu uma descentralização da gestão dos serviços em saúde, uma vez que os CCG, criados após as reformas do H\&SCA em 2012, assumiram maior autonomia para compra de serviços específicos para as suas necessidades regionais. No entanto, os fundos para esta aquisição continuaram advindos da instância centralizadora, o Departamento de Saúde do Reino Unido (DH). O DH deve financiar e regular a alocação dos recursos em saúde como um todo, incluindo, por exemplo, o repasse direto para os NHS dos países do Reino Unido, entretanto o NHS inglês é o que recebe a maior porcentagem de verba do DH e repassa quase $70 \%$ dos seus recursos para os CCG $(13,14)$.

Os critérios de repasse para os CCG são estabelecidos por uma ponderação per capita, ajustada a fatores epidemiológicos, socioeconômicos e desigualdades em saúde. Assim, o financiamento do sistema inglês se manteve concentrado na coleta de impostos da esfera nacional, diferente do SUS, onde as esferas estaduais e municipais contribuem para o financiamento do sistema com as suas arrecadações locais $(11,14,20,26)$.

Outro aspecto importante, em se tratando de financiamento em saúde, é a crescente participação do setor privado em ambos os sistemas. No SUS, entre 2002 e 2015 houve um aumento de $18 \%$ para $26 \%$ na taxa de contratações de planos de saúde privados pela população (26).

Isto se dá, basicamente, por três motivos principais: a) a saturação dos serviços públicos, levando usuários que têm recursos a buscar alternativas privadas; b) incentivos fiscais ao setor privado; c) incentivos à con- 
tratação dos serviços de saúde privado, como a PEC $n^{\circ} 451 / 2014$ (33), que propôs que a oferta do plano de assistência à saúde seja responsabilidade do empregador ao empregado $(19,26)$. No entanto, a participação do setor privado não é suficiente para desafogar o SUS, uma vez que ele só cobre aproximadamente $25 \%$ da população (16). O restante fica sob a responsabilidade exclusiva do SUS, configurando-o como o único sistema de saúde universal atualmente que cobre uma população de mais de 100 milhões de pessoas. Além disso, mesmo os $25 \%$ da população com acesso ao sistema de saúde suplementar acabam por utilizar o SUS quando o plano privado limita a cobertura $(20,34)$.

A Inglaterra, por sua vez, teve sua economia profundamente abalada após a crise financeira europeia vivida em 2009, o que fez com que o governo precisasse estabelecer políticas de cortes no NHS, estimando reduzir os gastos em saúde em 20 bilhões de libras entre 2010 e 2015 (13). Assim, uma reorganização das relações de custeio/aquisição e prestação de serviços foi estimulada, o que viria a acarretar uma intensificação da tendência às privatizações. Os CCG passaram a gerenciar cerca de $70 \%$ dos recursos do NHS inglês, ampliando suas responsabilidades dentro do sistema (13). Dentre elas, destaca-se a de contratação de serviços especializados e hospitalares, podendo adquiri-los de qualquer provedor, como de hospitais públicos do NHS ou do setor privado.

Ao mesmo tempo, seguindo a política de corte de custos, alguns hospitais públicos se tornaram autônomos podendo oferecer ao paciente a opção de pagamento direto pelo serviço prestado. Teve, assim, início da participação privada no NHS anteriormente menos evidenciada $(13,14)$.

No entanto, apesar do crescimento da iniciativa privada no sistema de saúde inglês, sua cobertura ainda é de $13 \%$ no país (13). Os gastos públicos em saúde no país permanecem superiores a $80 \%$ e a cobertura de serviços da população não foi afetada mesmo com a crise, mostrando que o sistema foi capaz de manter o seu caráter universal (13).

A crise econômica enfrentada pela Europa fez com que o NHS precisasse rever seus custos, aumentando a participação do mercado privado. As reformas recentes, aliadas à maior entrada de imigrantes no país nos últimos anos, levaram a população e os estudiosos a criticarem as mudanças efetuadas, temendo pelo fim da universalidade e acessibilidade do sistema, apontando, por exemplo, possíveis aumentos nos tempos de espera para cirurgias $(13,35)$.

\section{ATENÇÃO BÁSICA EM SAÚDE}

Sistema Único de Saúde. Segundo a OMS, a atenção básica remete a um conjunto de ações com intuito de melhorar, recuperar e manter a saúde de uma população $(16,36)$. Deve incluir cuidados a pacientes de todas as idades, condições socioeconômicas e origens geográficas. Profissionais da atenção básica são responsáveis por fornecer cuidados a uma gama de agravos à saúde de intensidades agudas e crônicas, desordens mentais e questões sociais de saúde (16,37).

No Brasil, a Atenção Básica em Saúde também seguiu a linha de descentralização, firmando as doutrinas do SUS. O Pacto pela Saúde (38), assinado em 2006, intensificou o processo de regionalização da saúde, organizando o sistema na forma de redes. A Portaria 4279/2010 (38) e o Decreto 7508/2011 (39) buscaram reestruturar a organização do sistema de saúde, estabelecendo, assim, as Redes de Atenção à Saúde (RAS) (11). A Portaria 4279/2010 (38) definiu a RAS como sendo "[...] arranjos organizativos de ações e serviços de saúde de diferentes densidades tecnológicas, que integradas por meio de sistemas de apoio técnico, logístico e de gestão, buscam garantir a integralidade do cuidado [...]" (38).

Ao mesmo tempo, no Brasil, foi estabelecida a Política Nacional de Atenção Básica (PNAB) (17) a fim de reforçar a atenção básica como porta de entrada e centro de comunicação das Redes de Atenção à Saúde. Seguindo a política do Ministério da Saúde de aprimoramento da atenção básica no país, a Política Nacional de Atenção Básica (PNAB) busca estabelecer serviços públicos de saúde mais acessíveis e com melhor qualidade. A PNAB, pretendeu estimular os gestores locais do SUS a fornecer uma melhor assistência aos usuários das suas Unidades Básicas de Saúde (UBS). Ainda, a PNAB incluiu as Equipes de Atenção Básica (EAB) para a população de rua e aumentou o número de municípios que poderiam ter Núcleos de Apoio à Saúde da Família (NASF) (17).

De modo geral, a Atenção Básica no Brasil é fornecida dentro das Unidades Básicas de Saúde (UBS), locais situados na região onde o paciente trabalha, estuda e vive. Nelas, o paciente é incluso no sistema, dá início ao seu tratamento e tem consultas regulares, recebendo acompanhamento de medicamentos e vacinas. É muito comum encontrar unidades de Assistência Médica Ambulatorial (AMA) acopladas às UBS, que são destinadas a enfermidades inesperadas, como febre, dor de cabeça, etc. (40). Nos AMA, o paciente não precisa agendar consulta, funcionando como pronto-socorro para sanar 
casos de baixa complexidade sem riscos de morte ou lesão irreversível.

Outros três serviços existentes no SUS e que cabem ser destacados são: Assistência Médica Ambulatorial de Especialidades (AMA-E), pertencente ao município e que fornece serviços secundários a pacientes encaminhados por uma UBS; Ambulatório Médico de Especialidades (AME), provido pelo Governo do Estado e que fornece serviços mais especializados, como exames e pequenas cirurgias; e Unidades de Pronto Atendimento (UPA) as quais auxiliam os prontos-socorros dos hospitais, constituindo locais de atuação $24 \mathrm{~h}$ por dia, 7 dias por semana, para serviços de urgência e emergência $(41,42)$.

Além disso, o programa Estratégia Saúde da Família (ESF) (43) se mostra como o principal modelo de atenção básica do Brasil. Nele, um morador do território abrangido pela Unidade de Saúde da Família entra para a equipe de saúde próxima à dos outros moradores do bairro e se torna Agente Comunitário de Saúde (ACS). Os ACS têm a função de transmitir conhecimento em saúde, de forma simplificada, à população pela qual eles estão responsáveis (43). Além dos ACS, no ESF a equipe multiprofissional (equipe de Saúde da Família - eSF) é composta por: médico generalista, ou especialista em Saúde da Família, ou médico de Família e Comunidade; enfermeiro generalista ou especialista em Saúde da Família; auxiliar ou técnico de enfermagem. A esta equipe, também podem ser adicionados profissionais de Saúde Bucal, como: cirurgião-dentista generalista ou especialista em Saúde da Família; auxiliar e/ou técnico em Saúde Bucal. É recomendado que cada eSF seja responsável por uma média 3000 pessoas, sendo aceito um máximo de 4000 pessoas por eSF. Além disso, deve ser levado em consideração o grau de vulnerabilidade das famílias na contagem do número de pessoas por eSF, devendo, assim, ser reduzido o número de pessoas se maior o grau de vulnerabilidade das famílias abrangidas (34).

Contudo, apesar de muito bem descrita nas diretrizes, portarias, e decretos, a Atenção Básica no Brasil ainda encontra dificuldades, principalmente, em questão de acessibilidade, apresentando, até 2012, apenas 30$40 \%$ da população com acesso à saúde básica de qualidade no país (15).

Apesar de constituir um modelo inovador de Atenção Básica, na prática as ESF encontram problemas para serem adequadamente estruturadas, em parte devidos ao baixo preparo técnico dos ACS e em parte às limitações dos ACS dentro das USF.
Cordeiro e Soares (2015) mostraram que cursos de formação para os ACS para melhorar a qualificação técnica, elevar o grau de escolaridade e aumentar as chances de melhor remuneração estavam previstos para ocorrerem desde 2004, porém, até 2010 ainda não haviam passado do primeiro módulo (de um total de três módulos) (43). Ainda, foi relatado que os ACS encontravam entraves e falta de espaço nas decisões dentro das USF.

Na prática, as decisões em saúde ainda se encontram centradas na figura do médico, havendo dificuldades da formação de uma equipe multiprofissional focada na elaboração de projetos em saúde-doença. Assim, não se tem resultado efetivo na solução dos problemas que acometem à população, uma vez que as necessidades das famílias muitas vezes não estão alinhadas com as indicações e protocolos pré-estabelecidos pelas unidades de saúde.

Pelo princípio da universalidade, todos os brasileiros têm direito aos serviços do SUS, e esse acesso universal, em nenhuma circunstância, pode ser restringido. O SUS deve ofertar, a todos os brasileiros, um conjunto de serviços sanitária e socialmente necessários, com base em Protocolos Clínicos e Diretrizes Terapêuticas, e por meio de amplo movimento de discussão que envolva os gestores de saúde na Comissão Intergestores Tripartite e o Conselho Nacional de Saúde (22).

Todos os municípios devem organizar uma atenção básica que seja resolutiva e que faça encaminhamentos responsáveis e adequados aos demais níveis de assistência. Considerando as diversidades demográficas e econômicas entre os municípios brasileiros, há que se salientar que a sua grande maioria não tem população suficiente que justifique a implantação de uma rede completa de serviços em todos os níveis hierárquicos (44).

Para possibilitar a montagem desta rede de serviços à saúde, é necessário integrar toda a oferta do setor público e incorporar serviços em caráter complementar, quando houver insuficiência ou inexistência na rede pública. Desta maneira, a Programação Pactuada e Integrada - PPI constitui-se em importante recurso capaz de instrumentalizar e apontar a quantidade e a diversidade de serviços a serem contratados, a sua localização e os fluxos de referência e contrarreferência (45).

Um estudo feito pela Fundação Oswaldo Cruz mostrou que, nas cidades brasileiras com menos de $30 \mathrm{mil}$ habitantes, é mais frequente a internação de pessoas por causa de enfermidades que podem ser prevenidas pela atenção básica $(46,47)$. São cidades muitas vezes distantes dos grandes centros urbanos, que arrecadam poucos 
impostos e onde os serviços de atenção básica à saúde falham, além da dificuldade de os municípios menos populosos fazerem uma estruturação adequada dos componentes da Assistência Farmacêutica. Como consequência, mais da metade dos municípios brasileiros estão nesta situação impactando, então, no encaminhamento de pacientes da atenção básica para outras cidades sobrecarregando, assim, os centros com maior infraestrutura de atendimento $(46,47)$.

National Health Service. Na Inglaterra, por sua vez, desde a criação do NHS em 1948, o sistema girou em torno da figura dos denominados General Practitioners (GP), médicos de atenção primária que atuavam como porta de entrada para a assistência hospitalar não emergencial. Cada paciente podia estar ligado a apenas um GP e cada GP recebia de acordo com o número de pacientes que possuía. Com o decorrer dos anos e a evolução do NHS, foram criados os Primary Care Trust (PCT), formados por médicos ou grupos de médicos da atenção primária que recebiam recursos dos Escritórios Regionais (12) do NHS para investir em serviços secundários. Esses grupos podiam comprar serviços de diferentes níveis de complexidade, podendo reter o excedente para melhoria de infraestrutura e dos serviços prestados aos pacientes (12).

Após a instituição do Health and Social Care Act (H\&SCA) em 2012 (13), os PCT foram substituídos pelos Clinical Commissioning Groups (CCG), que também assumiram a responsabilidade de planejamento, contratualização (commissioning), compra de serviços e estabelecimento de contratos com os provedores de atenção especializada. O NHS assumiu a função de autorização, contratação e monitoramento dos CCG, repasse de recursos e comissionamento de serviços de atenção primária e parte da atenção especializada de maior complexidade. O NHS, assim, se aproximou da função de financiamento dos serviços de saúde, deixando a função de prestação de serviços a cargo dos provedores por ele contratados, o que se refletiu também na atenção primária $(13,14)$.

Mais ainda, no que diz respeito à oferta de serviços de atenção primária, o NHS iniciou um processo de encorajamento das CCG pela contratação destes serviços. Por meio do NHS Five Year Forward View (24) lançado pelo governo após o H\&SCA, foi de interesse aumentar a autonomia das CCG na atenção primária buscando atender as necessidades regionais específicas e diminuir as desigualdades (24).

Duas outras importantes inovações que auxiliam no fortalecimento da atenção básica e, também, estão sob a responsabilidade do NHS foram os NHS Walk-In Centres e o NHS Direct $(12,14)$. Os NHS Walk-In Centres são centros abertos todos os dias da semana, de manhã à noite, sem necessidade de agendamento que oferecem serviços menos complexos e cuja avaliação é feita por enfermeiros. Estes centros complementam o trabalho dos GP, desafogando as clínicas médicas. O NHS Direct se constitui em linha telefônica com funcionamento $24 \mathrm{~h}$ na qual os enfermeiros fornecem informações de saúde e fazem o encaminhamento apropriado. Assim, há uma leve descentralização da saúde, conferindo certa autonomia a outros profissionais, processo este ainda fraco no SUS $(12,14,48)$.

Desta forma, em nível operacional, assim como no Brasil, a atenção básica na Inglaterra também sofreu um processo de regionalização, estando os provedores, contratados diretamente pelo NHS ou pelos CCG. No entanto, desde a existência do NHS, a Inglaterra buscou o fortalecimento da atenção básica se adaptando aos diferentes contextos econômicos e históricos pelo qual enfrentava, diferente do SUS, no qual as políticas para melhorar efetivamente a atenção básica foram publicadas tardiamente. Em relação à compra de recursos para a aquisição da atenção básica, ambos os sistemas mostraram uma descentralização da esfera nacional para as esferas regionais. No entanto, na Inglaterra essa transferência se deu de forma mais gradual, acompanhada de um aporte de conhecimentos e monitoramento da esfera nacional para a regional, diferente do Brasil onde, muitas vezes, os gestores regionais não possuem qualificação suficiente para gerir a saúde nas suas regiões $(11,13,17,49)$.

As criações das CCG apontam para um início da descentralização das responsabilidades do sistema, colocando o NHS em uma posição de provedor de saúde sem, necessariamente, garantir a acessibilidade dela.

Além do médico, as responsabilidades na prestação dos serviços em saúde no NHS são distribuídas, também, para outros profissionais, como enfermeiros e farmacêuticos. Desta forma, na Inglaterra os pacientes apresentam outras figuras na atenção primária, o que reflete diretamente na cobertura da atenção básica do país que, mesmo após as reformas, ainda se mantem universal.

\section{ACESSO A MEDICAMENTOS}

Sistema Único de Saúde. O âmbito da assistência farmacêutica tem ganhado destaque nas discussões internacionais a fim de melhorar o acesso a medicamentos 
pelas populações, garantia inclusa nos direitos humanos (50).

No SUS, com o estabelecimento da Política Nacional de Medicamentos (51) e da Política Nacional de Assistência Farmacêutica (52), intensificaram-se as discussões acerca da importância da Assistência Farmacêutica como componente crucial para garantia da atenção básica. Por meio dela, fortaleceu-se a ideia de que é essencial o estabelecimento de gestões que promovam o uso racional de medicamentos, garantam acesso a medicamentos considerados essenciais para a população e assegurem sua qualidade, segurança e eficácia.

Assim, a fim de se estabelecer medidas para viabilizar o acesso a medicamentos pelo SUS, a Assistência Farmacêutica foi dividida em três blocos de financiamento que são denominados componentes básico (de baixa complexidade), estratégico (apresentam algo impacto socioeconômico), e especializado (medicamentos e/ou tecnologias para doenças complexas) $(53,54)$.

Seguindo a política de descentralização intensificada após o Pacto pela Saúde em 2006 (38), no âmbito da Assistência Farmacêutica também foram definidas responsabilidades para os gestores das instâncias federais, estaduais e municipais. Com isso, buscava adequar a disponibilidade de medicamentos às reais necessidades das populações municipais, especialmente em relação aos medicamentos do Componente Básico da Assistência Farmacêutica. No entanto, na prática, este processo ainda encontra entraves para ser efetivamente realizado, devido à resistência dos médicos em aderir ao elenco de medicamentos disponíveis, à alta rotatividade dos profissionais, e à qualificação dos gestores municipais que, muitas vezes, ainda dependem da ajuda das Secretarias de Estado da Saúde (SES) para a elaboração de um plano de compra e manutenção de medicamento pautado em aspectos epidemiológicos e históricos de consumo $(53,54)$.

Além dos medicamentos fornecidos diretamente pelo SUS, o governo buscou criar programas para promover o melhor acesso pela população, como o Programa Farmácia Popular do Brasil (53). Por meio dele, o governo federal utilizou o potencial de farmácias privadas para disponibilizar medicamentos vendidos a preço de custo (53). O programa focava em medicamentos de uso contínuo, com intuito de impedir a interrupção do tratamento pelo paciente. Ao todo eram 25 medicamentos, sendo 14 deles $100 \%$ gratuitos e o restante com descontos de até $90 \%$. A maioria dos pacientes cobertos pelo programa (7,5 milhões) conseguia medicamentos gratuitamente, sendo a maior parte para tratamento de hipertensão (6,4 milhões) e diabetes (2,7 milhões) (55). O programa sofreu modificação em 2017 quando o Ministério da Saúde resolveu, em 30 de março, por meio da Comissão Intergestores Tripartie (CIT) encerrar o Programa Farmácia Popular - Rede Própria (PFP-RP), decorrene da parceria entre o Governo Federal e municípios que possuía 112 tipos de medicamentos essenciais. Os medicaments do elenco, segundo o Ministério da Saúde, poderão ser adquiridos nos postos de saúde municipais que terão uma ampliação de verba enviada aos municípios para compra de medicamentos, passando de $\mathrm{R} \$ 5,10 /$ habitante/mês para $\mathrm{R} \$ 5,58 /$ habitante/mês, valor que talvez não atenda as necessidades atuais dos municípios brasileiros (56). Portanto, o que continua funcionando, em rede privada, é o Programa Aqui Tem Farmácia Popular do Brasil com redução do elenco de medicamentos.

A disponibilização de recursos é fundamental para a assistência à saúde em todos os seus níveis. Entretanto, um problema importante que deve ser ressaltado é a judicialização de medicamentos nas diferentes esferas administrativas (32). A judicialização se refere à intervenção do Poder Judiciário no Poder Executivo a fim de obrigá-lo a realizar políticas públicas deficientes por meio de uma ordem judicial. Na saúde, a judicialização está relacionada à ação direta do Judiciário, como última alternativa, para a aquisição do medicamento ou tratamento negado pelo SUS, tanto devido a questões orçamentárias, quanto devido à ausência na lista da Relação Nacional de Medicamentos - RENAME (57).

No Brasil, o aumento da judicialização tem deixado gestores e juristas apreensivos, uma vez que pode levar a uma instabilidade orçamentária e afetar políticas públicas já avançadas. Este processo mostra, portanto, uma falha do sistema de saúde que tem se tornado incapaz de atender os princípios previstos constitucionalmente (58).

Reportagem em jornal aborda estudo que analisou 77 mil condenações sofridas pelo Governo do Estado de São Paulo entre 2010 e 2016 e evidenciou que 1 em cada 4 medicamentos ofertados por decisão da Justiça já estavam no SUS (59). O número de processos disparou nos últimos 5 anos (92\%) sendo que o Estado de São Paulo gasta por ano $\mathrm{R} \$ 1,2$ bilhão para atender 51 mil condenações e, segundo o texto da reportagem, o valor era o dobro do gasto no programa de terapias de alto custo da SES, que tinha 700 mil pacientes cadastrados além de que a justiça, também, obriga a comprar mais de 80 
itens que não são medicamentos para pessoas doentes ((59). Para enfrentar a questão da judicialização da saúde no plano nacional, o Ministério da Saúde firmou uma parceria com o Conselho Nacional de Justiça. A pasta financiará um banco de dados com notas técnicas e jurisprudência para embasar juízes (59).

Segundo o Ministério da Saúde, entre 2010 e 2014 os valores gastos para atender as decisões judiciais subiram $500 \%$, sendo boa parte destas ações referentes a tratamentos já previstos pelo SUS (60).

A promoção da saúde está sendo reconhecida cada vez mais como um elemento essencial para o desenvolvimento da saúde. É um processo para permitir que as pessoas tenham maior controle sobre sua saúde, e para melhorá-la. Mediante investimentos e ações, atua sobre os determinantes da saúde para criar o maior beneficio para os povos, para contribuir de maneira significativa para a redução das iniquidades em questões de saúde, para assegurar os direitos humanos e para a formação do capital social. A saúde é um direito humano fundamental e essencial para o desenvolvimento social e econômico. Não há sustentabilidade em um povo que não tenha saúde. O direito a saúde se confunde com o direito à vida (1).

National Health Service. Na Inglaterra, por sua vez, no âmbito da assistência farmacêutica, foi adotada a política de cofinanciamento dos medicamentos (61). Nela, os pacientes pagam um preço fixo $(£ 8,60)$ por cada item da prescrição, podendo optar por pagamentos trimestrais $(£ 29,10)$ e anuais $(£ 104,00)$ fixos, estes últimos vantajosos no caso de uso de mais de 3 medicamentos prescritos em 3 meses ou mais 12 em 1 ano, respectivamente. No entanto, alguns critérios podem tornar o paciente elegível à isenção da taxa de prescrição, tendo seu tratamento inteiramente gratuito (por exemplo: pacientes menores de 16 anos, idosos maiores de 60 anos, estudantes integrais entre 16-18 anos, grávidas ou mulheres que tiveram filhos nos últimos 12 meses, internados, entre outros) $(61,62)$.

Além dos critérios de isenção de prescrição, alguns medicamentos também podem ser obtidos gratuitamente por meio da utilização de serviços (61):

- Medicamentos administrados em hospitais e Walk-In-Centres.
- Contraceptivos

- Medicamentos administrados diretamente pelo GP ou via Grupos de Ajuda de Pacientes

- Medicamentos fornecidos por hospitais ou CCG para tratamento de doenças sexualmente transmissíveis, tuberculose ou para o tratamento de transtornos mentais a pessoas sujeitas a uma supervisão comunitária.

Os medicamentos isentos de prescrição (over-the-counter) tem comercialização liberada, podendo ser vendidos inclusive em supermercados, diferente do SUS, onde a comercialização é permitida somente em estabelecimentos autorizados. Ainda, na Inglaterra, há um forte incentivo à utilização de medicamentos genéricos a fim de melhorar o acesso a medicamentos pela população, ponto semelhante ao SUS (53).

Por fim, na Inglaterra as Farmácias Comunitárias exercem um papel crucial no tratamento dos pacientes. Nelas, os pacientes possuem uma relação próxima com o farmacêutico, buscando orientações sobre seu tratamento, recebendo orientações sobre uso racional de medicamentos e participando de consultas farmacêuticas, em casos tanto de novo tratamento quanto de tratamento contínuo, para fins de acompanhamento. Para pacientes em uso de medicamentos contínuos, adotou-se o processo de repeat prescription, onde a farmácia recebe, dos GP cadastrados, a prescrição dos pacientes e dispensa a quantidade exata para um período determinado, contendo orientações de uso. Ao final do período, a farmácia recebe uma nova prescrição e disponibiliza nova quantidade para o próximo período. Dessa forma, o tratamento do paciente é inteiramente monitorado tanto pelo farmacêutico quanto pelo médico (GP). Há, portanto, maior articulação entre o médico e o farmacêutico no tratamento do paciente, processo esse ainda fraco no SUS, onde o tratamento ainda é centrado na figura do médico $(53,62)$.

\section{RESUMO DAS PRINCIPAIS SEMELHANÇAS E DIFERENÇAS ENTRE SUS E NHS}

Os dois sistemas apresentam semelhanças e diferenças e o Quadro 1 resume os aspectos de maior importância neste sentido. 
Quadro 1. Principais semelhanças e diferenças entre o Sistema Único de Saúde (SUS), Brasil, e o National Health System (NHS), Reino Unido.

\begin{tabular}{|c|c|c|}
\hline & SUS & NHS (inglês) \\
\hline Doutrinas & $\begin{array}{l}\text { Universalidade } \\
\text { Equidade } \\
\text { Integralidade }\end{array}$ & $\begin{array}{l}\text { Universalidade } \\
\text { Equidade } \\
\text { Integralidade }\end{array}$ \\
\hline População atendida & Aproximadamente 207 milhões de pessoas & Aproximadamente 54 milhões de pessoas \\
\hline Gasto do PIB & $8,3 \%$ do PIB do Brasil & $\begin{array}{l}9,1 \% \text { do PIB do Reino Unido } \\
87 \% \text { repassado para o NHS inglês }\end{array}$ \\
\hline Financiamento & $\begin{array}{l}\text { Coleta de Imposto } \\
\text { Aumento da participação estadual e municipal (Descentralização) }\end{array}$ & $\begin{array}{l}\text { Coleta de Imposto } \\
\text { Repasse da verba da esfera nacional para as unidades } \\
\text { regionais por um critério per capita }\end{array}$ \\
\hline Participação setor privado & $\begin{array}{l}\text { Crescimento da participação privada } \\
\text { Percentual de participação privada: } 25 \%\end{array}$ & $\begin{array}{l}\text { Crescimento da participação privada } \\
\text { Percentual de participação privada: } 13 \%\end{array}$ \\
\hline Atenção Primária & $\begin{array}{l}\text { Prioridade recente } \\
\text { Cobertura abaixo de 50\% (dados de 2012) }\end{array}$ & $\begin{array}{l}\text { Prioridade do sistema desde a criação do NHS } \\
\text { Cobertura efetiva }\end{array}$ \\
\hline Equipe Multiprofissional & $\begin{array}{l}\text { Estabelecida em documentos oficiais } \\
\text { Fraco na prática } \\
\text { Saúde centrada no médico }\end{array}$ & $\begin{array}{l}\text { Estabelecido em documentos oficiais } \\
\text { Forte na prática } \\
\text { Enfermeiros e farmacêuticos possuem papel de destaque }\end{array}$ \\
\hline Acesso a medicamentos & $\begin{array}{l}\text { Medicamentos e tratamentos por meio dos Componentes Básico, Es- } \\
\text { tratégico e Especializado } \\
\text { Programa Farmácia Popular } \\
\text { Incentivo a genéricos }\end{array}$ & $\begin{array}{l}\text { Cofinanciamento } \\
\text { Isenção de Prescrições } \\
\text { Incentivo a genéricos }\end{array}$ \\
\hline Principal problema atual & $\begin{array}{l}\text { Falta de qualificação dos gestores regionais } \\
\text { Diminuição da participação federal na gestão e financiamento do SUS }\end{array}$ & $\begin{array}{l}\text { Crise europeia de } 2009 \\
\text { Corte de gastos do sistema }\end{array}$ \\
\hline
\end{tabular}

\section{CONCLUSÃO}

A contextualização aqui descrita mostrou que o gerenciamento de um sistema de saúde universal necessita de gestores com boa habilidade administrative, para cumprir a sua proposta inicial. Mesmo sistemas de saúde mais antigos e estruturados estão sujeitos a passar por situações de adaptações bruscas em decorrência do contexto social, político e econômico que enfrentam; entretanto, a capacidade administrativa dos gestores, se adequada, pode definir medidas benéficas à população, mesmo em situação de crise.

\section{REFERÊNCIAS}

1. Bastos ILG. O direito à saúde um desafio do SUS. 2009. Disponível em: http://www.webartigos.com/artigos/o-direito-a-saude-um-desafio-do-sus/26806/

2. Arruda VD. Relacionamento com stakeholders: Estudo em um laboratório na área de saúde humana. Pedro Leopoldo. Dissertação [Mestrado Profissional em Administração]. Fundação Pedro Leopoldo; 2015.

3. Nunes E. Principais sistemas de saúde no mundo. Disponível em: http://auditsaude.com.br/Sistemas\%20de\%20 Sa\%C3\%BAde\%20no\%20Mundo\%202.pdf

4. Pivetta SL. Direito fundamental à saúde. Regime jurídico, políticas públicas e controle judicial. São Paulo: Editora Revista dos Tribunais; 2014.

5. Declaração Universal dos Direitos Humanos. Aprovada em 10 de dezembro de 1948, em Paris. Disponível em: http://www.direitoshumanos.usp.br.
6. BRASIL. Constituição (1988). Constituição da República Federativa do Brasil. Brasília, DF: Senado Federal; 1988.

7. BRASIL. Presidência da República. Casa Civil. Subchefia para Assuntos Jurídicos. Lei n ${ }^{\circ} 8.080$, de 19 de setembro de 1990. Dispõe sobre as condições para a promoção, proteção e recuperação da saúde, a organização e o funcionamento dos serviços correspondentes e dá outras providências. Disponível em: http://www.planalto.gov. br/ccivil_03/leis/18080.htm

8. BRASIL. Ministério da Saúde. Lei 8.142, de 28 de dezembro de 1990. Dispõe sobre a participação da comunidade na gestão do Sistema Único de Saúde (SUS \} e sobre as transferências intergovernamentais de recursos financeiros na área da saúde e dá outras providências. Disponível em: http://conselho.saude.gov.br/legislacao/ lei8142 281290.htm 
9. Pinto-Júnior VL, Cerbino-Neto J, Penna GO. The evolution of the federal funding policies for the public health surveillance component of Brazil's Unified Health System (SUS). Ciênc Saúde Coletiva 2014; 19(12):48414849. DOI: $10.1590 / 1413-812320141912.05962013$

10. Pontes APM, Oliveira DC, Gomes AMT. Os princípios do Sistema Único de Saúde estudados a partir da análise de similitude. Rev Latino-Am Enfermagem 2014; 22(1):1-9. DOI: 10.1590/0104-1169.2925.2395

11. Machado CV, Lima LD, Baptista T.F. Princípios organizativos e instâncias de gestão do SUS. In: Oliveira RG, Grabois V, Mendes-Júnior WV, organizadores. Qualificação de gestores do SUS. Rio de Janeiro: EAD, Ensp; 2009.

12. Tanaka OY, Oliveira VE. Reforma(s) e estruturação do Sistema de Saúde Britânico: lições para o SUS. Saúde Soc 2007; 16(1):7-17.

13. Giovanella L, Stegmüller K. The financial crisis and health care systems in Europe: universal care under threat? Trends in health sector reforms in Germany, the United Kingdom, and Spain. Cad Saúde Pública 2014; 30(11):1-19. DOI: 10.1590/0102-311X00021314

14. NHS. National Health Service - England. Manual: Understanding the new NHS - A guide for everyone working and training within the NHS. Londres: BMJ; 2014. $26 \mathrm{p}$.

15. Santos NR. SUS, política pública de Estado: seu desenvolvimento instituído e instituinte e a busca de saídas. Ciênc Saúde Coletiva 2012; 18(1):273-280. DOI: 10.1590/S1413-81232013000100028

16. Costa KS, Soeiro OM, Nascimento-Júnior JM. As redes de Atenção à Saúde. In: Brasil. Ministério da Saúde. Secretaria de Ciência, Tecnologia e Insumos Estratégicos. Departamento de Assistência Farmacêutica e Insumos Estratégicos. Cuidado farmacêutico na Atenção Básica. Brasília: Ministério da Saúde; 2015. (Caderno 1: Serviços Farmacêuticos na Atenção Básica).

17. BRASIL. Ministério da Saúde. Portaria n. 2488, de 21 de outubro de 2011. Aprova a Política Nacional de Atenção Básica, estabelecendo a revisão de diretrizes e normas para a organização da Atenção Básica, para a Estratégia Saúde da Família (ESF) e o Programa de Agentes Comunitários de Saúde (PACS). Disponível em: http://bvsms.saude.gov.br/bvs/saudelegis/gm/2011/ prt2488_21_10_2011.html

18. BRASIL. Ministério da Saúde. Secretaria de Ciência, Tecnologia e Insumos Estratégicos. Departamento de Assistência Farmacêutica e Insumos Estratégicos. Cuidado Farmacêutico na atenção básica. Brasília: Ministério da Saúde; 2015. 4v.

19. Nóbrega CBC, Hoffmann RHS, Pereira AC, Meneghim MC. Financiamento do setor saúde: uma retrospectiva recente com uma abordagem para a odontologia. Ciênc Saúde Coletiva 2010; 15(1):1763-1772. DOI: 10.1590/ S1413-81232010000700088
20. BRASIL. Ministério da Saúde. Secretaria Nacional de Assistência à Saúde. ABC do SUS - doutrinas e princípios: manual de orientação. Brasília: Ministério da Saúde - Secretaria Nacional de Assistência à Saúde; 1990. 10p. (Séries Manuais).

21. BRASIL. Presidência da República. Decreto n. 8.065, de 7 de agosto de 2013. Aprova a estrutura regimental e o quadro demonstrativo dos cargos em comissão e das funções gratificadas do Ministério da Saúde e remaneja cargos em comissão. Disponível em: http://www.planalto. gov.br/ccivil_03/_Ato2011-2014/2013/Decreto/D8065. htm

22. BRASIL. Conselho Nacional de Secretários de Saúde CONASS. Sistema Único de Saúde.1.ed. Brasília: CONASS; 2011. (Coleção Para Entender a Gestão do SUS).

23. UUNITED KINGDOM. Health and Social Care Act 2012. Disponível em: http://www.legislation.gov.uk/ ukpga/2012/7/contents/enacted

24. UNITED KINGDOM. National Health Service (NHS Choices). The NHS in England. [legislation in Internet]. 2016. Disponível em: http://www.nhs.uk/NHSEngland/ thenhs/about/Pages/overview.aspx e https:/www.nhs.uk/ NHSEngland/thenhs/about/Pages/nhsstructure.aspx

25. Davies DSC. Developing the Public Health System. [Letter]. 2012 Abr 04. 2f. Disponível em: https:/www.gov. uk/government/publications/developing-the-new-public-health-system

26. Vieira FS. Implicações de decisões e discussões recentes para o financiamento do Sistema Único de Saúde. Saúde em Debate. 2016; 40(109):187-199. DOI: 10.1590/01031104201610915

27. WHO. World Health Organization. Countries. Disponível em: http://www.who.int/countres/bra/en

28. Lottenberg C. Saúde e cidadania: a tecnologia a serviço do paciente e não ao contrário. São Paulo: Editora Atheneu; 2015.

29. BRASIL. Presidência da República. Casa Civil. Subchefia para Assuntos Jurídicos. Emenda Constitucional $n^{\circ}$ 29, de 13 de setembro de 2000. Disponível em: http:// www.planalto.gov.br/ccivil_03/constituicao/emendas/ emc/emc29.htm

30. Saúde pública do país sofre de má gestão. O Globo. Disponível em: https://oglobo.com/opiniao/saude-publica-do-pais-sofre-de-ma-gestao-12010246\#ixzz50OIICalb. Postado em 28 de março de 2014.

31. Leal E. O cenário do desperdício de medicamentos no Brasil. 2014 Jun 24. Disponível: http://www.sensorweb.com.br/blog/desperdicio-medicamentos-brasil

32. Mapelle-Júnior R. Judicialização da saúde e políticas públicas: assistência farmacêutica, integralidade e regime jurídico constitucional do SUS. Tese [Doutorado em Ciências] - Faculdade de Medicina da Universidade de São Paulo; 2015. 
33. Cunha E. PEC 451/2000. Proposta de Emenda à Constituição. Disponível em: http://www.camara.gov.br/proposicoesWeb/fichadetramitacao?idProposicao $=861000$

34. BRASIL. Ministério da Saúde. Portal da Saúde. Estratégia Saúde da Família [documento na Internet]. 2012. Disponível em: http://dab.saude.gov.br/portaldab/ape_esf. php

35. Lionis C, Papadakaki M, Saridaki A, Dowrick C, O'Donnell CA, Mair FS, Van Den Muijsenbergh M, Burns N, Brún T, O’Reilly-De Brún M, Van Weel-Baumgarten E, Spiegel W, Macfarlane A. Engaging migrants and other stakeholders to improve communication in cross-cultural consultation in primary care: a theoretically informed participatory study. BMJ Open 2016 Jun; 6(7):2-15. DOI: 10.1136/bmjopen-2015- 010822

36. Mendes EV. As redes de aençãoà saúde. Ciênc Saúde Coletiva. 2010;15(5):2297-2305.

37. Calitri R, Adams A, Atherton H, Reeve J, Hill NR. Investigating the sustainability of careers in academic primary care: a UK survey. BMC Fam Pract 2014; 15(205):1-9. DOI: $10.1186 / \mathrm{s} 12875-014-0205-6$

38. BRASIL. Ministério da Saúde. Portaria n. 4.279, de 30 de dezembro de 2010. Estabelece diretrizes para a organização da Rede de Atenção à Saúde no âmbito do Sistema Único de Saúde (SUS). Disponível em: http://bvsms.saude.gov.br/bvs/saudelegis/gm/2010/ prt4279_30_12_2010.html

39. BRASIL. Presidência da República. Decreto $n^{\circ} 7.508$, de 28 de junho de 2011. Regulamenta a Lei n 8.080, de 19 de setembro de 1990. Dispõe sobre a organização do Sistema Único de Saúde - SUS, o planejamento da saúde, a assistência à saúde e a articulação interfederativa, e dá outras providências. Disponível em: http://www.planalto.gov.br/ ccivil_03/_ato2011-2014/2011/decreto/D7508.htm

40. SÃO PAULO. Prefeitura de São Paulo. Secretaria Municipal da Saúde. AMA Especialidades - Diretrizes Técnicas.1.ed. São Paulo; 2009.

41. BRASIL. Ministério da Saúde. Núcleo Estadual no Rio de Janeiro (NERJ). Ministério da Saúde informa: qual é a diferença entre UBAS e UBS? Confira! [documento na Internet]. 2014. Disponível em: www.nerj.rj.saude.gov. br/internet/?p=2896

42. APDM. Associação Paulista para o Desenvolvimento da Medicina. Ambulatório Médico de Especialidades (AMES). Disponível em: https://www.spdm.org.br/onde-estamos/outras-unidades/ambulatorio-medico-de-especialidades-ame

43. Cordeiro L, Soares CB. Work Process in Primary Health Care: action research with Community Health Workers. Ciênc Saúde Coletiva 2015; 20(11):3581-3588. DOI: 10.1590/1413-812320152011.10332014

44. Lima LD. A coordenação federattiva do Sistema público de saúde no Brasil. In: FUNDAÇÃO OSWALDO CRUZ. A saúde no Brasil em 2030 - prospecção estratégica do sistema de saúde brasileiro: organização e ggestão o sistema de saúde [livro na Inernet]. Rio de Janeiro: Fiocruz/ IPEA/Ministério da Saúde/Secretaria de Assuntos Estratégicos da Presidência da República; 2013.

45. SES-SP. Secretaria de Estado da Saúde de São Paulo, Conselho de Secretários Municipais de Saúde de São Paulo (COSEMS SP). SUS no estado de São Paulo. Atualizações para os gestores municipais. São Paulo: Polobooks; 2013. 206p

46. Ciscati R. Nos municípios pequenos, as pessoas sofrem mais com problemas de saúde evitáveis. Época, abr 11 2017. Disponível em http://epoca.globo.com/saude/noticia/2017/04/nos-municipios-pequenos-pessoas-sofrem-mais-com-problemas-de-saude-evitaveis.html

47. Pennafort R. Mais da metade dos pacientes precisa sair de suas cidades para fazer exames no Brasil. O Estado de São Paulo. Ago 26 2015. Disponível em http://brasil. estadao.com.br/noticias/geral,mais-da-metade-dos-pacientes-precisa-sair-de-suas-cidades-para-fazer-exames-no-brasil,1750647

48. Maier CB, Aiken LH. Task shifting from physicians to nurses in primary care in 39 countries: a cross-country comparative study. Eur J Public Health 2016; 26(6):927934. DOI: 10.1093/eurpub/ckw098

49. RCGP. Royal College of General Practioners. College campaign triggers major NHS England announcement on general practice. 2016 Abr 21 Disponível em: http:// www.rcgp.org.uk/news/2016/april/college-campaign-triggers-major-nhs-england-announcement-on-general-practice.aspx

50. Hunt P, Khosla R. Acesso a medicamentos como um direito humano. Sur Rev Int direitos human 2008; 5(8):101121. DOI: $10.1590 / \mathrm{S} 1806-64452008000100006$

51. BRASIL. Ministério da Saúde. Portaria ${ }^{\circ} 3.916$, de 30 de outubro de 1998. Aprova a Política Nacional de Medicamentos. Disponível em: bvsms.saude.gov.br/bvs/ saudelegis/gm/1998/prt/3916_30_10_1998.html

52. BRASIL. Conselho Nacional de Saúde. Resolução $n^{\circ}$ 338, de 06 de maio de 2004. Aprova a Política Nacional de Assistência Farmacêutica e estabelece seus princípios gerais e eixos estratégicos. Disponível em: bvsms.saude. gov.br/bvs/saudelegis/cns/2004/res0338_06_052004.html

53. BRASIL. Conselho Nacional de Secretários de Saúde (CONASS). O desafio do acesso a medicamentos nos Sistemas Públicos de Saúde: Relatório do Seminário Internacional de Assistência Farmacêutica do CONASS. Brasília: CONASS; 2010. 108p. (CONASS Documenta, n.20). Disponível em: http://www.conass.org.br/conassdocumenta/cd_20.pdf

54. Costa KS, Nascimento-Júnior JM, Soeiro OM, Paganelli MO, Araújo SQ. Assistência farmacêutica nas Redes de Atenção à Saúde do SUS. In: Brasil. Ministério da Saúde. Secretaria de Ciência, Tecnologia e Insumos Estratégicos. Departamento de Assistência Farmacêutica e 


\section{Ungarma}

Insumos Estratégicos. Cuidado farmacêutico na Atenção Básica. Brasília: Ministério da Saúde; 2015. (Caderno 1: Serviços Farmacêuticos na Atenção Básica).

55. BRASIL. Ministério da Saúde. Portal Brasil, Saúde. Aqui Tem Farmácia Popular atende 38 milhões de brasileiros em 10 anos. Disponível em: http://www.brasil. gov.br/saude/2016/03/aqui-tem-farmacia-popular-atende-38-milhoes-de-brasileiros-em-10-anos

56. Maciel V. Agência Saúde. Atendimento à imprenssa. Ascom/MS. Saúde altera repasse do Farmácia Popular e amplia em R\$ 80 milhões por ano recurso para medicamentos. Disponível em: http://portalsaude.saude.gov.br/ index.php/cidadao/principal/agencia-saude/28642-nova-forma-de-repasse-amplia-em-r-80-milhoes-por-ano-recurso-para-oferta-de-medicamentos Postado em jun 06; modificado em jun 08 .

57. BRASIL. Ministério da Saúde. Secretaria de Ciência, tecnóloga e Insumos Estratégicos. Departamento de Assistência Farmacêutica e Insumos Estratégicos. Relação Nacional de Medicamentos Essenciais: RENAME 2017/ Ministério da Saúde, Secretaria de Ciência, Tecnologia e Insumos Estratégicos, Departamento de Assistência Farmacêutica e Insumos Estratégicos. Brasília: Ministério da Saúde; 2017.
58. Silva LC. Judicialização da saúde: em busca de uma contenção saudável. Âmbito Jurídico 2013;16(112). Disponível em: http://www.ambitojuridico.com.br/site/?n link=revista_artigos_leitura\&artigo_id=13182\&revista_caderno $=9$

59. Pinho A. 1 em 4 remédios ofertados por decisão da Justiça já está no SUS. Folha de São Paulo 2016 Set 24; Caderno Cotidiano B1, B1, B3 e B4.

60. Brito R. Judicialização da Saúde deve aumentar gastos em R\$ 7 bilhões. 2016 Jun 28 [revista na Internet]. Disponível em: http://exame.abril.com.br/economia/noticias/judicializacao-da-saude-deve-aumentar-gastos-em-r-7-bilhoes

61. UNITED KINGDOM. National Health Service (NHS Choices). NHS in England - help with health costs. 2016 Abr 11. Disponível em: http://www.nhs.uk/NHSEngland/Healthcosts/Pages/Prescriptioncosts.aspx

62. UNITED KINGDOM. National Health Service (NHS Choices). NHS pharmacy services explained. 2015 nov. Disponível em: http://www.nhs.uk/NHSEngland/AboutNHSservices/pharmacists/Pages/pharmacistsandchemists.aspx. 